\title{
Clique Partitions of Glued Graphs
}

\author{
Chariya Uiyyasathian(Corresponding author) \\ Department of Mathematics, Faculty of Science \\ Chulalongkorn University, Bangkok 10330, Thailand \\ Tel: 66-2-218-5159 E-mail: Chariya.U@Chula.ac.th \\ Uthoomporn Jongthawonwuth \\ Department of Mathematics, Faculty of Science \\ Chulalongkorn University, Bangkok 10330, Thailand \\ Tel: 66-81-792-0936 E-mail: aor_utoo@hotmail.com
}

The research is partially financed by the DPST (Development and Promotion of Science and Technology Talents Project, Thailand).

\begin{abstract}
A glued graph at $K_{2}$-clone ( $K_{3}$-clone) results from combining two vertex-disjoint graphs by identifying an edge (a triangle) of each original graph. The clique covering numbers of these desired glued graphs have been investigated recently. Analogously, we obtain bounds of the clique partition numbers of glued graphs at $K_{2}$-clones and $K_{3}$-clones in terms of the clique partition numbers of their original graphs. Moreover, we characterize glued graphs satisfying such bounds.
\end{abstract}

Keywords: Clique partitions, Clique coverings, Glued graphs

\section{Introduction}

Let $G_{1}$ and $G_{2}$ be any two graphs with disjoint vertex sets. Let $H_{1}$ and $H_{2}$ be nontrivial connected subgraphs of $G_{1}$ and $G_{2}$, respectively, such that $H_{1} \cong H_{2}$ with an isomorphism $f$. We combine $G_{1}$ and $G_{2}$ by identifying $H_{1}$ and $H_{2}$ with respect to the isomorphism $f$. This resulting graph is called glued graph of $G_{1}$ and $G_{2}$ at $H_{1}$ and $H_{2}$ with respect to $f$. We denote this glued graph by $G_{1} \triangleleft G_{2}$ where $H$ is the copy of $H_{1}$ and $H_{2}$ in the glued graph. We refer to $H, H_{1}$ and $H_{2}$ as the clones of the glued graph, $G_{1}$ and $G_{2}$, respectively, and refer to $G_{1}$ and $G_{2}$ as the original graphs. Thus the combined graph is also called the glued graph of $G_{1}$ and $G_{2}$ at $H$-clone The notation $G_{1} \triangleleft G_{2}$ represents an arbitrary graph resulting from gluing graphs $G_{1}$ and $G_{2}$ at any isomorphic subgraph $H_{1} \cong H_{2}$ with respect to any of their isomorphism. More details concerning glued graphs can be explored in Promsakon's thesis (Promsakon, 2006). Furthermore, planar glued graphs and perfection of glued graphs are also discussed recently (see (Uiyyasathian and Hemakul, 2006) and (Saduakdee and Uiyyasathian, 2009).

Cliques are complete subgraphs of a graph that are not necessarily maximal. An $n$-clique or a clique of order $n$ of a graph $G$ is a clique of $G$ with $n$ vertices. A clique covering of a graph $G$ is a set of cliques of $G$, which together contain each edge of $G$ at least once; if each edge is covered exactly once, then it is called clique partition. The clique covering number $c c(G)$ and clique partition number $c p(G)$ are the smallest cardinality among all clique coverings and clique partitions of $G$, respectively. A minimum clique partition of $G$ is a clique partition of $G$ with cardinality $c p(G)$. Since a clique partition of a graph $G$ is also a clique covering of $G$, we always have $c c(G) \leq c p(G)$.

$G-H$ denotes the graph derived from $G$ obtained by deleting all edges of a subgraph $H$. In particular, if $e$ is an edge of $G$, then $G-e$ is the graph $G$ with the edge $e$ deleted. The notation $e(G)$ stands for the number of edges in a graph $G$.

The question of calculating clique covering and clique partition numbers was raised by Orlin in 1977. DeBruijn and Erdős had already proved that partitioning a complete graph $K_{n}$ into smaller cliques required at least $n$ cliques (DeBruijn \& Erdôs, 1948). This yields the following theorem of Orlin which states that for $n \geq 3, c p\left(K_{n}-e\right)=n-1$ where $e$ is any edge of the complete graph $K_{n}$ (Orlin, 1977). A recent paper by Pimpasalee et al. (Pimpasalee et al., 2008), investigates bounds of clique covering numbers of glued graphs at $K_{2}$-clones as follows:

$$
c c\left(G_{1}\right)+c c\left(G_{2}\right)-1 \leq c c\left(\underset{K_{2}}{G_{1}} G_{2}\right) \leq c c\left(G_{1}\right)+c c\left(G_{2}\right) .
$$

They also give a characterization of glued graphs with the clique covering number of each possible value. This paper investigates analogously the clique partition numbers of glued graphs in terms of their original graphs.

Note first that a glued graph can possibly have its clique partition number less than the clique partition number of each original graph, see Example A.

Example A Let $G_{1}$ be a Hamiltonian graph on $n$ vertices with a Hamiltonian path $P$ as shown in the bold edges in Figure 1. and $G_{2}=\bar{G}_{1} \cup P$. Then the resulting graph $\underset{P}{G_{1} \triangleleft G_{2}}$ is a complete graph, so $\operatorname{cp}\left(\underset{P}{\left.G_{1} \triangleleft G_{2}\right)}\right)=1$. Since neither $G_{1}$ nor $G_{2}$ 
is a complete graph, its clique partition number is more than 1. It is noticeable that the graph gluing of original graphs with any arbitrary large clique partition number could yield a resulting glued graph with clique partition number 1 . This situation occurs because of the existence of new cliques in the glued graphs.

Consequently, we observed that if $G_{1} \triangleleft G_{2}$ does not have a new clique, at least $c p\left(G_{1}\right)\left(\right.$ and $\left.c p\left(G_{2}\right)\right)$ cliques are needed to partition the copy of $G_{1}$ (and $G_{2}$ ) in $G_{1} \triangleleft G_{2}$. Hence,

$$
c p\left(G_{1} \triangleleft G_{2}\right) \geq \max \left\{c p\left(G_{1}\right), c p\left(G_{2}\right)\right\} .
$$

Back to clique coverings of arbitrary glued graphs, since a set of union between minimum clique coverings of both original graphs is a clique covering of such glued graph (see Pimpasalee et al., 2008), $c c\left(G_{1} \triangleleft G_{2}\right) \leq c c\left(G_{1}\right)+c c\left(G_{2}\right)$. However, the same situation is not true for clique partition numbers, see Example B.

Example B Claim that $c p\left({ }_{K_{2}} K_{n}\right)=\min \{m, n\}$ for $m, n \geq 3$.

Let $m \geq n \geq 3$. Since $K_{m}{ }_{K_{2}}^{\triangleleft} K_{n}$ can be partitioned into the sets of an $m$-clique, an $(n-1)$-clique and $(n-2)$ copies of 2-cliques, $c p\left(K_{K_{2}} K_{n}\right) \leq n$. Orlin proved that $c p\left(K_{n}-e\right)=n-1$ (Orlin, 1977) for $n \geq 3$ and $e$ is an edge of $K_{n}$. Since $n \leq m$, we obtain that $c p\left(K_{m} \bowtie K_{n}\right)>n-1$. Thus, $c p\left(K_{K_{2}} K_{n}\right)=n$. Note that $c p\left(K_{m}\right)+c p\left(K_{n}\right)=2$ while $c p\left(K_{m} \triangleleft K_{n}\right)$ can be arbitrary large. It is an evident that a graph gluing even at the smallest clone may use much more cliques to partition our resulting glued graphs than those of original graphs.

This leads us to consider a possible upper bound for the clique partition numbers of arbitrary glued graphs. For any glued graphs of $G_{1}$ and $G_{2}$ at a clone $H$, since the union of a minimum clique partition of $G_{1}$ (or $G_{2}$ ) and minimum clique partition of $G_{2}-H$ (or $G_{1}-H$ ) provides a clique partition of $\underset{H}{G_{1} \triangleleft G_{2}}$, it yields that

$$
c p\left(G_{1} G_{H}\right) \leq \min _{\substack{i, j=1,2 \\ i \neq j}}\left\{c p\left(G_{i}\right)+c p\left(G_{j}-H\right)\right\} .
$$

Our main results focus on the graph gluing at $K_{2}$-clone and $K_{3}$-clone. We give some characterizations of glued graphs with the clique partition numbers of some possible values.

\section{Preliminaries}

For convenience, throughout the rest of the paper, we refer $K_{n}$ in the glued graph $G_{1}{ }_{K_{n}} G_{2}$ to be only the $K_{n}$-clone, not an arbitrary copy of $K_{n}$ in our graphs. In a clique partition, if an edge $e$ is covered by a 2 -clique itself, $e$ is also refered to as such a clique. Moreover, we will also refer $E(G)$ to be a set of 2-cliques.

Definition 1 Let $\mathscr{P}$ be a minimum clique partition of a glued graph $G_{1} \triangleleft G_{2}$. We define $\mathscr{P}\left[G_{1}\right]=\left\{C \in \mathscr{P} \mid C\right.$ is a clique of $\left.G_{1}\right\}$ and $\mathscr{P}\left[G_{2}\right]=\left\{C \in \mathscr{P} \mid C\right.$ is a clique of $\left.G_{2}\right\}$.

Note from the Definition 1 that $\mathscr{P}\left[G_{1}\right] \cup \mathscr{P}\left[G_{2}\right] \subseteq \mathscr{P}$. However $G_{1} \stackrel{\leftrightarrow}{H} G_{2}$ contains a new clique $C$ if and only if $C \notin$ $\mathscr{P}\left[G_{1}\right] \cup \mathscr{P}\left[G_{2}\right]$. Any glued graph at $K_{n}$-clone does not have a new clique, so we have the following result:

Proposition 2 For a minimum clique partition $\mathscr{P}$ of $\underset{K_{n}}{G_{1} \triangleleft G_{2}, \mathscr{P}}=\mathscr{P}\left[G_{1}\right] \cup \mathscr{P}\left[G_{2}\right]$.

The known results about an edge deletion or $n$-clique deletion on the clique partition number are conclued next. Theorems 3-7 help us to investigate bounds of clique partition numbers of glued graphs at $K_{2}$-clones and $K_{3}$-clones.

Theorem 3 (Monson, 1996) Let s be the order of the smallest clique containing the edge e among all of the minimum clique partitions of $G$. Then

$$
c p(G)-1 \leq c p(G-e) \leq c p(G)+s-2 .
$$

Theorem 4 (Pullman, 1981) For $n \geq 4, n-1 \leq c p\left(K_{n}-K_{3}\right) \leq 2 n-5$.

Theorem 5 For any graph $G$ and clique $C$ of $G, c p(G)-1 \leq c p(G-C)$, and the equality holds if and only if there exists a minimum clique partition of $G$ containing $C$.

Proof. Let $\mathscr{P}$ and $\mathscr{P}^{\prime}$ be minimum clique partitions of $G$ and $G-C$, respectively. Then $\mathscr{P}^{\prime} \cup\{C\}$ is a clique partition of $G$, so $\operatorname{cp}(G) \leq|\mathscr{P} \cup\{C\}|$ and hence $c p(G)-1 \leq c p(G-C)$.

If every minimum clique partition of $G$ does not contain $C$, then $\mathscr{P}^{\prime} \cup\{C\}$ cannot be a minimum clique partition of $G$. So, $\left|\mathscr{P}^{\prime} \cup\{C\}\right|>|\mathscr{P}|$. Hence, $c p(G-C)+1=\left|\mathscr{P}^{\prime} \cup\{C\}\right|>|\mathscr{P}|=c p(G)$.

Suppose that $\mathscr{P}$ contains $C$. Then $\mathscr{P} \backslash\{C\}$ is a clique partition of $G-C$. Thus, $\operatorname{cp}(G-C) \leq|\mathscr{P}|-1=c p(G)-1$. Therefore, the equality holds.

www.ccsenet.org/jmr 
Theorem 6 Let $G_{1}$ and $G_{2}$ be graphs containing $K_{n}$ as a subgraph. If there exists a minimum clique partition of $G_{1}$ or $G_{2}$ containing the $K_{n}$-clone, then $c p\left(\underset{K_{n}}{G_{1} \triangleleft G_{2}}\right) \leq c p\left(G_{1}\right)+\operatorname{cp}\left(G_{2}\right)-1$

Proof. It follows immediately from equation (1) and Theorem 5.

Lemma 12 If there exists a minimum clique partition of $\underset{K_{n}}{G_{1} \triangleleft G_{2}}$ containing the $K_{n}$-clone, then there exists a minimum clique partition of $G_{1}$ or $G_{2}$ containing the $K_{n}$-clone.

Proof. Let $\mathscr{P}$ and $\mathscr{P}_{1}$ be minimum clique partitions of $G_{1} \bowtie G_{2}$ containing the $K_{n}$-clone and $G_{1}$, respectively. Since the glued graph at $K_{n}$-clone has no new cliques, $\mathscr{P}\left[G_{1}\right]$ and $\mathscr{P}\left[G_{2}\right]$ are clique partitions of $G_{1}$ and $G_{2}$, respectively. Let $\mathscr{P}{ }_{1}$ be a minimum clique partition of $G_{1}$. Note that $\mathscr{P}=\left(\mathscr{P}\left[G_{1}\right] \backslash\left\{K_{n}\right\}\right) \cup\left(\mathscr{P}\left[G_{2}\right] \backslash\left\{K_{n}\right\}\right) \cup\left\{K_{n}\right\}$. Now, suppose that all minimum clique partitions of $G_{1}$ and $G_{2}$ do not contain the $K_{n}$-clone. Then $\mathscr{P}_{1}$ does not contain the $K_{n}$-clone. Since $\mathscr{P}\left[G_{1}\right]$ is a clique partition of $G_{1}$ containing the $K_{n}$-clone, $\left|\mathscr{P}\left[G_{1}\right]\right|>\left|\mathscr{P}_{1}\right|=c p\left(G_{1}\right)$. Consequently, $\left|\mathscr{P}\left[G_{1}\right] \backslash\left\{K_{n}\right\}\right| \geq c p\left(G_{1}\right)$. Thus,

$$
|\mathscr{P}|=\left|\mathscr{P}\left[G_{1}\right] \backslash\left\{K_{n}\right\}\right|+\left|\mathscr{P}\left[G_{2}\right] \backslash\left\{K_{n}\right\}\right|+1 \geq c p\left(G_{1}\right)+\left|\mathscr{P}\left[G_{2}\right] \backslash\left\{K_{n}\right\}\right|+1 .
$$

Observe that $\mathscr{P}_{1} \cup\left(\mathscr{P}\left[G_{2}\right] \backslash\left\{K_{n}\right\}\right)$ is also a clique partition of $G_{1}{ }_{K_{n}} G_{2}$ and $\left|\mathscr{P} 1 \cup\left(\mathscr{P}\left[G_{2}\right] \backslash\left\{K_{n}\right\}\right)\right|=\operatorname{cp}\left(G_{1}\right)+\left|\mathscr{P}\left[G_{2}\right] \backslash\left\{K_{n}\right\}\right|$, this contradicts the minimality of $\mathscr{P}$.

Theorem 7 If there exists a minimum clique partition of $\underset{K_{n}}{G_{1} \triangleleft G_{2}}$ containing the $K_{n}$-clone, then

$$
c p\left(\underset{K_{n}}{G_{1}} G_{2}\right)=c p\left(G_{1}\right)+c p\left(G_{2}\right)-1 .
$$

Proof. Let $\mathscr{P}$ be a minimum clique partition of $G_{1} \triangleleft G_{2}$ containing the $K_{n}$-clone. By Proposition $2, \mathscr{P}=\mathscr{P}\left[G_{1}\right] \cup \mathscr{P}\left[G_{2}\right]$. Since $\mathscr{P}$ contains the $K_{n}$-clone, $\mathscr{P}\left[G_{1}\right] \cap \mathscr{P}\left[G_{2}\right]=\left\{K_{n}\right\}$. Note that $\mathscr{P}\left[G_{1}\right]$ and $\mathscr{P}\left[G_{2}\right]$ are clique partitions of $G_{1}$ and $G_{2}$, respectively,so $\left|\mathscr{P}\left[G_{1}\right]\right| \geq c p\left(G_{1}\right)$ and $\left|\mathscr{P}\left[G_{2}\right]\right| \geq c p\left(G_{2}\right)$. Thus,

$$
\begin{aligned}
c p\left({ }_{K_{n}}^{G_{1} \triangleleft G_{2}}\right)=|\mathscr{P}| & =\left|\mathscr{P}\left[G_{1}\right] \cup \mathscr{P}\left[G_{2}\right]\right| \\
& =\left|\mathscr{P}\left[G_{1}\right]\right|+\left|\mathscr{P}\left[G_{2}\right]\right|-\left|\mathscr{P}\left[G_{1}\right] \cap \mathscr{P}\left[G_{2}\right]\right| \\
& \geq c p\left(G_{1}\right)+c p\left(G_{2}\right)-1 .
\end{aligned}
$$

By Lemma 12, without loss of generality, $G_{1}$ has a minimum clique partition, $\mathscr{P}_{1}$, containing the $K_{n}$-clone. Let $\mathscr{P}_{2}$ be a minimum clique partition of $G_{2}$. Then $\left(\mathscr{P}_{1} \backslash\left\{K_{n}\right\}\right) \cup \mathscr{P}_{2}$ is a clique partition of $\underset{K_{n}}{G_{1} \triangleleft G_{2}}$. Therefore,

$$
c p\left(\begin{array}{c}
G_{1} \bowtie G_{2} \\
K_{n}
\end{array}\right) \leq\left|\left(\mathscr{P}_{1} \backslash\left\{K_{n}\right\}\right) \cup \mathscr{P}_{2}\right|=\left|\left(\mathscr{P}_{1} \backslash\left\{K_{n}\right\}\right)\right|+\left|\mathscr{P}_{2}\right|=c p\left(G_{1}\right)+c p\left(G_{2}\right)-1 .
$$

Hence, $c p\left(\underset{K_{n}}{G_{1} \bowtie G_{2}}\right)=c p\left(G_{1}\right)+c p\left(G_{2}\right)-1$.

\section{Clique Partitions of Glued Graphs at $K_{2}$-Clones}

Theorem 9 shows bounds of clique covering numbers of any glued graphs at $K_{2}$-clones. An analogous statement for clique partition numbers is studied in Theorem 10. Notice that the lower bound has the same style while the upper bound does not. Example B illustrates that $c p\left(G_{1}\right)+c p\left(G_{2}\right)$ is not an upper bound for the clique partition numbers of $\underset{K_{2}}{G_{1} \triangleleft G_{2}}$.

Remark 8 Let $\mathscr{P}$ be a minimum clique partition of ${ }_{K_{2}}^{G_{1} G_{2}}$.

(i) $\mathscr{P}\left[G_{1}\right] \cap \mathscr{P}\left[G_{2}\right] \subseteq\left\{K_{2}\right\}$.

(ii) If the $K_{2}$-clone is contained in $\mathscr{P}$, then $\mathscr{P}\left[G_{1}\right] \cap \mathscr{P}\left[G_{2}\right]=\left\{K_{2}\right\}$, and, $\mathscr{P}\left[G_{1}\right]$ and $\mathscr{P}\left[G_{2}\right]$ are clique partitions of $G_{1}$ and $G_{2}$, respectively.

(iii) If the $K_{2}$-clone is not contained in $\mathscr{P}$, then $\mathscr{P}\left[G_{1}\right] \cap \mathscr{P}\left[G_{2}\right]=\emptyset$, furthermore, if the clone $K_{2} \in \mathscr{P}\left[G_{i}\right] \backslash \mathscr{P}\left[G_{j}\right]$ for some $i \neq j \in\{1,2\}$, then $\mathscr{P}\left[G_{i}\right]$ and $\mathscr{P}\left[G_{j}\right]$ are clique partitions of $G_{i}$ and $G_{j}-K_{2}$, respectively.

Theorem 9 (Pimpasalee et al, 2008) For any graphs $G_{1}$ and $G_{2}$,

$$
c c\left(G_{1}\right)+c c\left(G_{2}\right)-1 \leq c c\left({ }_{K_{2}}^{G_{1} \bowtie G_{2}}\right) \leq c c\left(G_{1}\right)+c c\left(G_{2}\right) .
$$


Theorem 10 For any graphs $G_{1}$ and $G_{2}$,

$$
c p\left(G_{1}\right)+c p\left(G_{2}\right)-1 \leq c p\left({ }_{K_{2}}^{G_{1} \triangleleft G_{2}}\right) \leq c p\left(G_{1}\right)+c p\left(G_{2}\right)+s-2
$$

where $s$ is the order of the smallest clique containing the $K_{2}$-clone among all of the minimum clique partitions of $G_{1}$ and $G_{2}$.

Proof. Without loss of generality, we may assume that $G_{2}$ has a minimum clique partition containing clique of order $s$ containing the $K_{2}$-clone. By equation (1) and Theorem 3, $c p\left({ }_{K_{2}}^{G_{1}} G_{2}\right) \leq c p\left(G_{1}\right)+c p\left(G_{2}-K_{2}\right) \leq c p\left(G_{1}\right)+c p\left(G_{2}\right)+s-2$.

Now, let $\mathscr{P}$ be a minimum clique partition of $G_{K_{2}} G_{2}$. If $K_{2} \in \mathscr{P}$, then $c p\left({ }_{K_{2}}^{G_{1}} G_{2}\right)=c p\left(G_{1}\right)+c p\left(G_{2}\right)-1$. Suppose that $K_{2} \notin \mathscr{P}$. Then $\mathscr{P}$ is partitioned into $\mathscr{P}\left[G_{1}\right]$ and $\mathscr{P}\left[G_{2}\right]$. We may assume that $\mathscr{P}\left[G_{1}\right]$ and $\mathscr{P}\left[G_{2}\right] \cup\left\{K_{2}\right\}$ are clique partitions of $G_{1}$ and $G_{2}$, respectively. Therefore,

$$
c p\left(\underset{K_{2}}{G_{1} \triangleleft G_{2}}\right)=|\mathscr{P}|=\left|\mathscr{P}\left[G_{1}\right]\right|+\left|\mathscr{P}\left[G_{2}\right]\right|=\left|\mathscr{P}\left[G_{1}\right]\right|+\left|\mathscr{P}\left[G_{2}\right] \cup\left\{K_{2}\right\}\right|-1 \geq c p\left(G_{1}\right)+c p\left(G_{2}\right)-1 .
$$

Recall from Example B that $c p\left(K_{K_{2}} \triangleleft K_{n}\right)=\min \{m, n\}$. Note that for the glued graph $K_{m_{2}} \triangleleft K_{n}, s$ defined in Theorem 10 is $\min \{m, n\}$. Thus, $c p\left(K_{m}\right)+c p\left(K_{2}\right)+s-2=1+1+\min \{m, n\}-2=c p\left(K_{m}{ }_{K_{2}} K_{n}\right)$. Therefore, the upper bound in equation (3) is sharp.

A characterization of $G_{1} \bowtie G_{2}$ whose clique covering number satisfying the lower bound in equation (2) is investigated, see Pimpasalee et al. (Pimpasalee et al., 2008). Next, we reveal these analogous statements for glued graphs at $K_{2}$-clones with clique partition numbers satisfying the lower bound in equation (3).

Theorem 11 For any graphs $G_{1}$ and $G_{2}$, the following statements are equivalent:

(i) $c p\left(\underset{K_{2}}{G_{1} G_{2}}\right)=c p\left(G_{1}\right)+c p\left(G_{2}\right)-1$,

(ii) $G_{1}$ or $G_{2}$ has a minimum clique partition containing the $K_{2}$-clone, and

(iii) $c p\left(G_{1}-K_{2}\right)=c p\left(G_{1}\right)-1 \operatorname{or} c p\left(G_{2}-K_{2}\right)=c p\left(G_{2}\right)-1$.

Proof. (ii) $\Rightarrow$ (i) This follows directly from Theorems 6 and 10 .

(i) $\Rightarrow$ (iii) Assume that $c p\left(\underset{K_{2}}{G_{1} \triangleleft G_{2}}\right)=c p\left(G_{1}\right)+c p\left(G_{2}\right)-1$. Let $\mathscr{P}$ be a minimum clique partition of $G_{1} \triangleleft G_{2}$. If $K_{2} \in \mathscr{P}$, by Lemma 12, there exists a minimum clique partition of $G_{1}$ or $G_{2}$ containing the $K_{2}$-clone. Applying Theorem 5 yields $c p\left(G_{1}-K_{2}\right)=c p\left(G_{1}\right)-1$ or $c p\left(G_{2}-K_{2}\right)=c p\left(G_{2}\right)-1$. Assume that $K_{2} \notin \mathscr{P}$. Then $|\mathscr{P}|=\left|\mathscr{P}\left[G_{1}\right]\right|+\left|\mathscr{P}\left[G_{2}\right]\right|$. We may assume that $\mathscr{P}\left[G_{2}\right]$ is a clique partition of $G_{2}$, and then $\mathscr{P}\left[G_{1}\right]$ is a clique partition of $G_{1}-K_{2}$. Thus $c p\left(G_{1}\right)+c p\left(G_{2}\right)-1=$ $c p\left(G_{1} \bowtie G_{2}\right)=|\mathscr{P}|=\left|\mathscr{P}\left[G_{1}\right]\right|+\left|\mathscr{P}\left[G_{2}\right]\right| \geq c p\left(G_{1}-K_{2}\right)+c p\left(G_{2}\right)$, so $c p\left(G_{1}\right)-1 \geq c p\left(G_{1}-K_{2}\right)$. Again apply Theorem 3 to have $c p\left(G_{1}\right)-1=c p\left(G_{1}-K_{2}\right)$.

(iii) $\Rightarrow$ (ii) It follows immediately from Theorems 5 .

Corollary 13 If $c p\left(\begin{array}{c}G_{1} \bowtie G_{2} \\ K_{2}\end{array}\right)=c p\left(G_{1}\right)+c p\left(G_{2}\right)$, then $c p\left(G_{1}\right) \leq c p\left(G_{1}-K_{2}\right)$ and $c p\left(G_{2}\right) \leq c p\left(G_{2}-K_{2}\right)$.

Proof. It follows immediately from Theorems 3 and 11.

Corollary 14 follows directly from Lemma 12 and Theorem 11 .

Corollary 14 If there exists a minimum clique partition of $\underset{K_{2}}{G_{1} \triangleleft G_{2}}$ containing the $K_{2}$-clone, then

(i) $c p\left({ }_{K_{2}}^{G_{1} \triangleleft G_{2}}\right)=c p\left(G_{1}\right)+c p\left(G_{2}\right)-1$, or

(ii) $c p\left(G_{i}-K_{2}\right)=c p\left(G_{i}\right)-1$ for some $i \in\{1,2\}$.

For any graph $G$, the statement in Theorem 3, namely $c p(G-e) \geq c p(G)-1$, can be rewritten by $c p(G-e)=c p(G)+t$ for some $t \geq-1$. Next, we consider the clique partition number of a glued graph $G_{K_{2}} G_{2}$ satisfying $\operatorname{cp}\left(G_{i}-K_{2}\right)=c p\left(G_{i}\right)+t_{i}$ where $i \in\{1,2\}$. The special case when $t_{i}=-1$ for some $i \in\{1,2\}$ has been already examined in Theorem 11 . 
Theorem 12 Let $G_{1} \bowtie G_{2}$ be a glued graph at $K_{2}$-clone. If $c p\left(G_{1}-K_{2}\right)=c p\left(G_{1}\right)+t_{1}$ and $c p\left(G_{2}-K_{2}\right)=c p\left(G_{2}\right)+t_{2}$ for some integers $t_{1}, t_{2}$, then $c p\left(\underset{K_{2}}{G_{1} \triangleleft G_{2}}\right)=c p\left(G_{1}\right)+c p\left(G_{2}\right)+t$ where $t=\min \left\{t_{1}, t_{2}\right\}$.

Proof. First, by Theorem 3 we note that $t_{1}, t_{2} \geq-1$. If $t_{i}=-1$ for some $i \in\{1,2\}$, then the statement is true by Theorem 11 . Otherwise, assume that $0 \leq t_{1} \leq t_{2}$. Since a union of a minimum clique partition of $G_{1}-K_{2}$ and a minimum clique partition of $G_{2}$ is a clique partition of $G_{1} \triangleleft G_{2}, c p\left(G_{1} \triangleleft G_{2}\right) \leq c p\left(G_{1}\right)+c p\left(G_{2}\right)+t_{1}$. Let $\mathscr{P}$ be a minimum clique partition of $G_{1} \triangleleft G_{2}$. By Proposition $2, \mathscr{P}=\mathscr{P}\left[G_{1}\right] \cup \mathscr{P}\left[G_{2}\right]$. Since $t_{1}, t_{2} \geq 0$ and by Corollary 14 , the $K_{2}$-clone is not in $\mathscr{P}$ and then $\mathscr{P}$ is partitioned into $\mathscr{P}\left[G_{1}\right]$ and $\mathscr{P}\left[G_{2}\right]$. We have two cases.

Case 1. $\mathscr{P}\left[G_{1}\right]$ is a clique partition of $G_{1}$ and $\mathscr{P}\left[G_{2}\right]$ is a clique partition of $G_{2}-K_{2}$. Thus, $\operatorname{cp}\left(G_{1} \bowtie G_{2}\right)=|\mathscr{P}|=$ $\left|\mathscr{P}\left[G_{1}\right]\right|+\left|\mathscr{P}\left[G_{2}\right]\right| \geq c p\left(G_{1}\right)+c p\left(G_{2}\right)+t_{2} \geq c p\left(G_{1}\right)+c p\left(G_{2}\right)+t_{1}$. Hence, $c p\left({ }_{K_{2}}^{G_{1}} G_{2}\right)=c p\left(G_{1}\right)+c p\left(G_{2}\right)+t$ where $t=\min \left\{t_{1}, t_{2}\right\}$.

Case 2. $\mathscr{P}\left[G_{2}\right]$ is a clique partition of $G_{2}$ and $\mathscr{P}\left[G_{1}\right]$ is a clique partition of $G_{1}-K_{2}$. Thus, $\operatorname{cp}\left(G_{1} \bowtie G_{2}\right)=|\mathscr{P}|=$ $\left|\mathscr{P}\left[G_{1}\right]\right|+\left|\mathscr{P}\left[G_{2}\right]\right| \geq c p\left(G_{1}\right)+c p\left(G_{2}\right)+t_{1}$. Hence, $c p\left(\underset{K_{2}}{G_{1} \triangleleft G_{2}}\right)=c p\left(G_{1}\right)+c p\left(G_{2}\right)+t$ where $t=\min \left\{t_{1}, t_{2}\right\}$.

Now a characterization of $G_{1} \triangleleft G_{2}$ satisfying the upper bound in equation (2) which states that $\operatorname{cp}\left({ }_{K_{2}} G_{K_{2}} G_{2}\right)=\operatorname{cp}\left(G_{1}\right)+$ $c p\left(G_{2}\right)+s-2$ where $s \geq 3$ is obtained in the following theorem.

Theorem 13 Let $G_{1}{ }_{K_{2}} G_{2}$ be a glued graph of $G_{1}$ and $G_{2}$ at $K_{2}$-clone, and $s$ the order of the smallest clique containing the $K_{2}$-clone among all of the minimum clique partitions of $G_{1}$ and $G_{2}$ where $s \geq 3$. Then $c p\left(\underset{K_{2}}{G_{1} \triangleleft G_{2}}\right)=\operatorname{cp}\left(G_{1}\right)+c p\left(G_{2}\right)+s-2$ if and only if, $\operatorname{cp}\left(G_{i}-K_{2}\right) \geq \operatorname{cp}\left(G_{i}\right)+s-2$ for each $i \in\{1,2\}$.

Proof. Assume that $c p\left(\underset{K_{2}}{G_{1} \triangleleft G_{2}}\right)=c p\left(G_{1}\right)+c p\left(G_{2}\right)+s-2$. We have that $c p\left({ }_{K_{2}}^{G_{1} \triangleleft G_{2}}\right) \leq c p\left(G_{j}\right)+c p\left(G_{i}-K_{2}\right)$ for all $i \neq j \in\{1,2\}$. It follows that $c p\left(G_{i}-K_{2}\right) \geq c p\left(G_{i}\right)+s-2$ for all $i \in\{1,2\}$.

Conversely, assume that $c p\left(G_{i}-K_{2}\right) \geq c p\left(G_{i}\right)+s-2$ for all $i \in\{1,2\}$. Let $\mathscr{P}$ be a minimum clique partition of $G_{1} \triangleleft G_{2}$. By Proposition 2, P्P $=\mathscr{P}\left[G_{1}\right] \cup \mathscr{P}\left[G_{2}\right]$. Since $s \geq 3$ and by Corollary 14 , the $K_{2}$-clone is not in $\mathscr{P}$. Then $|\mathscr{P}|^{K_{2}}=\left|\mathscr{P}\left[G_{1}\right]\right|+\left|\mathscr{P}\left[G_{2}\right]\right|$. Without loss of generality, let $\mathscr{P}\left[G_{1}\right]$ be a clique partition of $G_{1}$. Then $\mathscr{P}\left[G_{2}\right]$ is a clique partition of $G_{2}-K_{2}$. Thus, $c p\left({ }_{K_{2}}^{G_{1}} G_{2}\right)=|\mathscr{P}|=\left|\mathscr{P}\left[G_{1}\right]\right|+\left|\mathscr{P}\left[G_{2}\right]\right| \geq c p\left(G_{1}\right)+c p\left(G_{2}-K_{2}\right) \geq c p\left(G_{1}\right)+c p\left(G_{2}\right)+s-2$. Together with Theorem 10, we have $c p\left(\underset{K_{2}}{G_{1} \triangleleft G_{2}}\right)=c p\left(G_{1}\right)+c p\left(G_{2}\right)+s-2$ as desired.

\section{Clique Partitions of Glued Graphs at $K_{3}$-Clones}

Pimpasalee obtains bounds of clique covering numbers of any glued graphs at $K_{3}$-clones as in Theorem 14 . We study these analogous statements for clique partition numbers in this section.

Theorem 14 (Pimpasalee, 2008) For any graphs $G_{1}$ and $G_{2}$ containing $K_{3}$ as a subgraph,

$$
c c\left(G_{1}\right)+c c\left(G_{2}\right)-2 \leq c c\left(\underset{K_{3}}{G_{1} \triangleleft G_{2}}\right) \leq c c\left(G_{1}\right)+c c\left(G_{2}\right) .
$$

Definition 15 Let $\mathscr{P}$ be a minimum clique partition of a glued graph $\underset{K_{n}}{G_{1} \triangleleft G_{2}}$. We define

$$
\begin{aligned}
& E_{1}[\mathscr{P}]=\left\{e \in E\left(K_{n}\right) \mid e \text { is not covered by any clique in } \mathscr{P}\left[G_{1}\right]\right\} \text { and } \\
& E_{2}[\mathscr{P}]=\left\{e \in E\left(K_{n}\right) \mid e \text { is not covered by any clique in } \mathscr{P}\left[G_{2}\right]\right\} .
\end{aligned}
$$

Note that $\mathscr{P}\left[G_{1}\right] \cup E_{1}[\mathscr{P}]$ and $\mathscr{P}\left[G_{2}\right] \cup E_{2}[\mathscr{P}]$ are clique partitions of $G_{1}$ and $G_{2}$, respectively.

Theorem 16 Let $G_{1}$ and $G_{2}$ be graphs containing $K_{3}$ as a subgraph. Then

$$
c p\left(G_{1}\right)+c p\left(G_{2}\right)-3 \leq c p\left(\underset{K_{3}}{G_{1} \triangleleft G_{2}}\right) .
$$

Proof. Let $\mathscr{P}$ be a minimum clique partition of $G_{1} \bowtie G_{2}$. Since the clone is a complete graph, $\mathscr{P}=\mathscr{P}\left[G_{1}\right] \cup \mathscr{P}\left[G_{2}\right]$. So, $\mathscr{P}=\left[\left(\mathscr{P}\left[G_{1}\right] \cup E_{1}[\mathscr{P}]\right) \cup\left(\mathscr{P}\left[G_{2}\right] \cup E_{2}[\mathscr{P}]\right)\right] \backslash\left(E_{1}[\mathscr{P}] \cup E_{2}[\mathscr{P}]\right)$. Thus,

$$
\begin{aligned}
c p\left(\underset{K_{3}}{G_{1} \triangleleft G_{2}}\right) & =|\mathscr{P}|=\left|\left[\left(\mathscr{P}\left[G_{1}\right] \cup E_{1}[\mathscr{P}]\right) \cup\left(\mathscr{P}\left[G_{2}\right] \cup E_{2}[\mathscr{P}]\right)\right] \backslash\left(E_{1}[\mathscr{P}] \cup E_{2}[\mathscr{P}]\right)\right| \\
& \left.\left.=\mid \mathscr{P}\left[G_{1}\right] \cup E_{1}[\mathscr{P}]\right)|+| \mathscr{P}\left[G_{2}\right] \cup E_{2}[\mathscr{P}]\right)|-|\left(\mathscr{P}\left[G_{1}\right] \cap \mathscr{P}\left[G_{2}\right]\right) \cup\left(E_{1}[\mathscr{P}] \cup E_{2}[\mathscr{P}]\right) \mid .
\end{aligned}
$$


Since $\left|\mathscr{P}\left[G_{1}\right] \cup E_{1}[\mathscr{P}]\right| \geq c p\left(G_{1}\right)\left|\mathscr{P}\left[G_{2}\right] \cup E_{2}[\mathscr{P}]\right| \geq c p\left(G_{2}\right)$ and $\left|\left(\mathscr{P}\left[G_{1}\right] \cap \mathscr{P}\left[G_{2}\right]\right) \cup\left(E_{1}[\mathscr{P}] \cup E_{2}[\mathscr{P}]\right)\right| \leq 3$, we have

$$
\begin{aligned}
c p\left({ }_{K_{3}}^{G_{1} \triangleleft G_{2}}\right) & \geq c p\left(G_{1}\right)+c p\left(G_{2}\right)-\left|\left(\mathscr{P}\left[G_{1}\right] \cap \mathscr{P}\left[G_{2}\right]\right) \cup\left(E_{1}[\mathscr{P}] \cup E_{2}[\mathscr{P}]\right)\right| \\
& \geq c p\left(G_{1}\right)+c p\left(G_{2}\right)-3 .
\end{aligned}
$$

Definition 17 Let $G$ be a graph containing a triangle $T$ and $\mathscr{P}$ a minimum clique partition of $G$. Then we say that

1. $\mathscr{P}$ is type 1 with respect to the triangle $T$, if $\mathscr{P}$ contains the triangle $T$;

2. $\mathscr{P}$ is type 2 with respect to the triangle $T$, if $\mathscr{P}$ contains a clique of order at least 4 covering the triangle $T$;

3. Otherwise, $\mathscr{P}$ is type 3 with respect to the triangle $T$, that is, each edge of the triangle $T$ is covered by different cliques in $\mathscr{P}$.

Remark 18 Let $G_{K_{3}}^{G_{1} G_{2}}$ be a glued graph at $K_{3}$-clone and $\mathscr{P}$ a minimum clique partition of $\underset{K_{3}}{G_{1} G_{2}}$.

(i) If $\mathscr{P}$ is type 1 with respect to the $K_{3}$-clone, then $\mathscr{P}\left[G_{1}\right] \cap \mathscr{P}\left[G_{2}\right]=\left\{K_{3}\right\}$, and hence, $\mathscr{P}\left[G_{1}\right]$ and $\mathscr{P}\left[G_{2}\right]$ are clique partitions of $G_{1}$ and $G_{2}$, respectively.

(ii) If $\mathscr{P}$ is type 2 with respect to the $K_{3}$-clone, then $\mathscr{P}\left[G_{1}\right] \cap \mathscr{P}\left[G_{2}\right]=\emptyset$, furthermore, $\mathscr{P}\left[G_{i}\right]$ and $\mathscr{P}\left[G_{j}\right]$ are clique partitions of $G_{i}$ and $G_{j}-K_{3}$, respectively, for some $i \neq j \in\{1,2\}$.

(iii) If $\mathscr{P}$ is type 3 with respect to the $K_{3}$-clone, then an element in $\mathscr{P}\left[G_{1}\right] \cap \mathscr{P}\left[G_{2}\right]$ is a proper subset of $E\left(K_{3}\right)$, consequently, $\left|\mathscr{P}\left[G_{1}\right] \cap \mathscr{P}\left[G_{2}\right]\right|=0,1$ or 2 .

Theorem 19 Let $G_{1} \bowtie G_{2}$ be a glued graph at $K_{3}$-clone. If $G_{1}{ }_{K_{3}} G_{2}$ has a minimum clique partition which is type 1 or type 2 with respect to the $K_{3}$-clone, then $c p\left(\underset{K_{3}}{G_{1} \triangleleft G_{2}}\right) \geq \operatorname{cp}\left(G_{1}\right)+c p\left(G_{2}\right)-1$.

Proof. Let $\mathscr{P}$ be a minimum clique partition of $\underset{K_{3}}{G_{1} \triangleleft G_{2}}$. By Proposition 2, $\mathscr{P}=\mathscr{P}\left[G_{1}\right] \cup \mathscr{P}\left[G_{2}\right]$.

Case 1. $\mathscr{P}$ is type 1 with respect to the $K_{3}$-clone. By Theorem $7, c p\left(\underset{K_{3}}{G_{1} \triangleleft G_{2}}\right)=c p\left(G_{1}\right)+c p\left(G_{2}\right)-1$.

Case 2. $\mathscr{P}$ is type 2 with respect to the $K_{3}$-clone. Without loss of generality, the clique of order at least 4 containing the $K_{3}$-clone is in $\mathscr{P}\left[G_{1}\right]$. Then $\mathscr{P}\left[G_{1}\right]$ and $\mathscr{P}\left[G_{2}\right] \cup\left\{K_{3}\right\}$ are clique partitions of $G_{1}$ and $G_{2}$, respectively. Thus,

$$
c p\left(\begin{array}{c}
G_{1} \bowtie G_{2} \\
K_{3}
\end{array}\right)=|\mathscr{P}|=\left|\mathscr{P}\left[G_{1}\right]\right|+\left|\mathscr{P}\left[G_{2}\right]\right|=\left|\mathscr{P}\left[G_{1}\right]\right|+\left|\mathscr{P}\left[G_{2}\right] \cup\left\{K_{3}\right\}\right|-1 \geq c p\left(G_{1}\right)+c p\left(G_{2}\right)-1 .
$$

Theorem 20 Let $G_{1} \bowtie G_{2}$ be a glued graph of $G_{1}$ and $G_{2}$ at $K_{3}$-clone. Then $c p\left({ }_{K_{1}}{ }_{K_{3}} G_{2}\right)=\operatorname{cp}\left(G_{1}\right)+\operatorname{cp}\left(G_{2}\right)-3$ if and only if there exist minimum clique partitions $\mathscr{P}_{1}$ and $\mathscr{P}_{2}$ of $G_{1}$ and $G_{2}$, respectively, such that for each edge e $\in E\left(K_{3}\right), e$ must be covered by a 2 -clique in $\mathscr{P}_{1}$ or $\mathscr{P}_{2}$.

Proof. First, assume that $c p\left(G_{1} \bowtie G_{2}\right)=c p\left(G_{1}\right)+c p\left(G_{2}\right)-3$. Let $\mathscr{P}$ be a minimum clique partition of $G_{K_{3}} G_{2}$. By Proposition $2, \mathscr{P}=\mathscr{P}\left[G_{1}\right] \cup \mathscr{P}\left[G_{2}\right]$. Note that

$$
|\mathscr{P}|=\left|\mathscr{P}\left[G_{1}\right] \cup E_{1}[\mathscr{P}]\right|+\left|\mathscr{P}\left[G_{2}\right] \cup E_{2}[\mathscr{P}]\right|-\left|\mathscr{P}\left[G_{1}\right] \cap \mathscr{P}\left[G_{2}\right]\right|-\left|E_{1}[\mathscr{P}] \cup E_{2}[\mathscr{P}]\right| .
$$

Moreover, we have that $|\mathscr{P}|=c p\left(G_{1}\right)+c p\left(G_{2}\right)-3, \mathscr{P}\left[G_{i}\right] \cup E_{i}[\mathscr{P}]$ is a clique partition of $G_{i}$ for all $i \in\{1,2\}$ and $\left|\mathscr{P}\left[G_{1}\right] \cap \mathscr{P}\left[G_{2}\right]\right|+\left|E_{1}[\mathscr{P}] \cup E_{2}[\mathscr{P}]\right| \leq 3$. Hence, $\left|\mathscr{P}\left[G_{i}\right] \cup E_{i}[\mathscr{P}]\right|=c p\left(G_{i}\right)$ for all $i \in\{1,2\}$ and $\left|\mathscr{P}\left[G_{1}\right] \cap \mathscr{P}\left[G_{2}\right]\right|+\left|E_{1}[\mathscr{P}] \cup E_{2}[\mathscr{P}]\right|=3$. Therefore $\mathscr{P}\left[G_{i}\right] \cup E_{i}[\mathscr{P}]$ is a minimum clique partition of $G_{i}$ for all $i \in\{1,2\}$. Let $e$ be an edge in the $K_{3}$-clone of $G_{1} \triangleleft G_{2}$. If $e \in \mathscr{P}\left[G_{1}\right] \cap \mathscr{P}\left[G_{2}\right]$, then $e$ is covered by a 2-clique in $\mathscr{P}\left[G_{1}\right]$ and $\mathscr{P}\left[G_{2}\right]$. Thus $e \in \mathscr{P}\left[G_{i}\right] \cup E_{i}[\mathscr{P}]$ for all $i \in\{1,2\}$. Suppose that $e \notin \mathscr{P}\left[G_{1}\right] \cap \mathscr{P}\left[G_{2}\right]$. Then there exists a clique $C$ of order more than two in $\mathscr{P}$ covering $e$. Without loss of generality, assume that $C \in \mathscr{P}\left[G_{1}\right]$. Then $e \in E_{2}[\mathscr{P}]$, so $e \in \mathscr{P}\left[G_{2}\right] \cup E_{2}[\mathscr{P}]$. For sufficiency, assume that $G_{1}$ and $G_{2}$ have minimum clique partitions $\mathscr{P}_{1}$ and $\mathscr{P}_{2}$, respectively, such that satisfy the condition in the right hand side of the statement. Let $A=\left\{e \in E\left(K_{3}\right) \mid e \in \mathscr{P}_{1}\right\}$ and $B=\left\{e \in E\left(K_{3}\right) \mid e \in \mathscr{P}_{2}\right\}$. Note 
that $|A|+|B|-|A \cap B|=e\left(K_{3}\right)=3$ and $\left(\mathscr{P}_{1} \backslash A\right) \cup\left(\mathscr{P}_{2} \backslash B\right) \cup(A \cap B)$ is a clique partition of $G_{1} \bowtie G_{2}$. Thus, $\left|\left(\mathscr{P}_{1} \backslash A\right) \cup\left(\mathscr{P}_{2} \backslash B\right) \cup(A \cap B)\right| \geq c p\left(\underset{K_{3}}{G_{1} \triangleleft G_{2}}\right)$. Hence,

$$
\begin{aligned}
c p\left(G_{1}\right)+c p\left(G_{2}\right)-3 & =\left|\mathscr{P}_{1}\right|+\left|\mathscr{P}_{2}\right|-|A|-|B|+|A \cap B| \\
& =\left|\left(\mathscr{P}_{1} \backslash A\right) \cup\left(\mathscr{P}_{2} \backslash B\right) \cup(A \cap B)\right| \\
& \geq c p\left(\begin{array}{c}
G_{1} \triangleleft G_{2} \\
K_{3}
\end{array}\right) .
\end{aligned}
$$

By Theorem 16, $c p\left(\underset{K_{3}}{G_{1} \triangleleft G_{2}}\right)=c p\left(G_{1}\right)+c p\left(G_{2}\right)-3$.

The following theorem gives the possible values of $c p\left(\underset{K_{3}}{G_{1} \triangleleft G_{2}}\right)$ when $G_{1}$ or $G_{2}$ has a minimum clique partition which is type 1 with respect to the $K_{3}$-clone.

Theorem 21 Let $G_{1} \bowtie G_{2}$ be a glued graph at $K_{3}$-clone. If $G_{1}$ or $G_{2}$ has a minimum clique partition which is type 1 with respect to the $K_{3}$-clone, then

$$
c p\left(G_{1}\right)+c p\left(G_{2}\right)-3 \leq c p\left(\underset{K_{3}}{G_{1}} G_{2}\right) \leq c p\left(G_{1}\right)+c p\left(G_{2}\right)-1 .
$$

Proof. It follows directly from Theorems 6 and 16.

Theorem 22 Let $\underset{K_{3}}{G_{1} \triangleleft G_{2}}$ be any graph at $K_{3}$-clone and $\mathscr{P}_{i}$ a minimum clique partition of $G_{i}$ for each $i \in\{1,2\}$. Then

$$
c p\left({ }_{K_{3}}^{G_{1}} \underset{G_{2}}{ }\right) \leq c p\left(G_{1}\right)+c p\left(G_{2}\right)-6+\min \left\{\sigma_{1}, \sigma_{2}\right\}
$$

where for each $i \in\{1,2\}, s_{i}$ is the sum of orders of all cliques in $\mathscr{P}_{i}$ containing edges of the $K_{3}$-clone, and

$$
\sigma_{i}= \begin{cases}2 s_{i} & \text { if } \mathscr{P}_{i} \text { is type } 2 \text { with respect to the } K_{3}-\text { clone } \\ s_{i} & \text { if } \mathscr{P}_{i} \text { is type } 3 \text { with respect to the } K_{3}-\text { clone }\end{cases}
$$

Proof. Assume that $\mathscr{P}_{1}$ and $\mathscr{P}_{2}$ are minimum clique partitions of $G_{1}$ and $G_{2}$, respectively.

Here we suppose that $\mathscr{P}_{1}$ and $\mathscr{P}_{2}$ are type 2 and type 3 with respect to the $K_{3}$-clone, respectively. For other cases, the proof follows similarly. Since $\mathscr{P}_{1}$ is type 2 with respect to the $K_{3}$-clone, there exists a clique $C \in \mathscr{P}_{1}$ with order $r \geq 4$ containing $K_{3}$. Then $G_{1}-K_{3}$ can be partitioned by the union of $\mathscr{P}_{1} \backslash\{C\}$ and a minimum clique partition of $C-K_{3}$. By Theorem 4, $c p\left(C-K_{3}\right) \leq 2 r-5$. Thus $c p\left(G_{1}-K_{3}\right) \leq\left|\mathscr{P}_{1}\right|-1+2 r-5=c p\left(G_{1}\right)+2 r-6$. Since $\mathscr{P}_{2}$ is type 3 with respect to the $K_{3}$-clone, there exists three cliques in $\mathscr{P}_{2}$ such that each one covers different edge in the $K_{3}$-clone, say $Q_{1}, Q_{2}$ and $Q_{3}$ of order $q_{1}, q_{2}$ and $q_{3}$, respectively. Then $G_{2}-K_{3}$ can be partitioned by the union of $\mathscr{P}_{2} \backslash\left\{Q_{1}, Q_{2}, Q_{3}\right\}$ and a minimum clique partition of $Q_{i}$ deleted an edge in the $K_{3}$-clone for all $i \in\{1,2,3\}$. We have that $\operatorname{cp}\left(Q_{i}-e_{i}\right)=q_{i}-1$ where $Q_{i}$ covers an edge $e_{i}$ in the $K_{3}$-clone for all $i \in\{1,2,3\}$. Thus,

$$
c p\left(G_{2}-K_{3}\right) \leq\left|\mathscr{P}_{2}\right|-3+\left(q_{1}-1\right)+\left(q_{2}-1\right)+\left(q_{3}-1\right)=c p\left(G_{2}\right)+q_{1}+q_{2}+q_{3}-6 .
$$

Hence, $c p\left(\underset{K_{3}}{G_{1} \triangleleft G_{2}}\right) \leq c p\left(G_{1}\right)+c p\left(G_{2}\right)-6+\min \left\{\sigma_{1}, \sigma_{2}\right\}$ where $\sigma_{1}=2 r$ and $\sigma_{2}=q_{1}+q_{2}+q_{3}$.

\section{An Application}

In general, the problem of computing clique partition numbers is considered to be difficult. Only for some classes of graphs can be obtained their clique partition numbers or even given reasonable bounds. The problem of determining the clique partition numbers of graphs is NP-complete, see Ma et al. (Ma et al., 1988). In an appropriate way, the glue operator could sometimes be used to reduce a large graph into smaller graphs which are easier to find their clique partition numbers than directly find the clique partition number of the large graph.

Example C Consider a graph $G$ shown in Figure 2. Here we illustrate the usefulness of the glue operator to find $c p(G)$. We refer a bold edge in $G$ to be the $K_{2}$-clone and reduce the graph $G$ into $G_{1}, G_{2}$ and $G_{3}$ as shown as in Figure 2 . Then $G \cong$ $\underset{K_{2}}{\left(G_{1} \triangleleft G_{2}\right) \triangleleft G_{3}}$. Note that $c p\left(G_{1}\right)=5, c p\left(G_{2}\right)=1$ and $c p\left(G_{3}\right)=3$. Since $G_{1}$ has a minimum clique partition containing the $K_{2}$-clone, by Theorem 10, $c p\left({ }_{K_{2}}^{G_{1} \triangleleft G_{2}}\right)=c p\left(G_{1}\right)+c p\left(G_{2}\right)-1=5+1-1=5$. Similarly, $G_{3}$ has a minimum clique partition containing the $K_{2}$-clone, and again by Theorem 10, $c p\left(\underset{K_{2}}{\left(G_{1} \underset{K_{2}}{\triangleleft} G_{2}\right) \triangleleft G_{3}}\right)=c p\left(\underset{K_{2}}{G_{1} \triangleleft G_{2}}\right)+c p\left(G_{3}\right)-1=5+3-1=7$. Hence, $c p(G)=7$. 


\section{References}

Bruijn de N.G. and Erdős P. (1948). On a combinatorial problem, Indag. Math., 10, 421-423

Ma S., Wallis W. D. and Wu J. (1988). On the complexity of the clique partition problem, Congr. Numer., 67, 59-66.

Monson S. D. (1996). The Effects of vertex deletion and edge deletion on the Clique Partition Number, Ars. Combinatoria., 42, 89-96.

Orlin J. (1977). Contentment in graph theory: covering graphs with cliques, Indag. Math., 39, 406-424.

Pimpasalee W. (2008). Clique Coverings of Glued Graphs. Master Degree Thesis, Chulalongkorn University, Thailand.

Pimpasalee W., Hemakul W. and Uiyyasathian C. (2008). Clique coverings of glued graphs at the clones $K_{2}$. Proceedings of the Thirteenth Annual Meeting in Mathematics 2008, Srinakharinwirot University, Thailand, 39-44.

Promsakon C. (2006). Colorability of Glued Graphs. Master Degree Thesis, Chulalongkorn University, Thailand.

Pullman N. J. and Donald A. (1981). Clique Coverings of Graphs II: Complements of Cliques. Utilitas Math., 19, 207-213.

Saduakdee S. and Uiyyasathian C. (2009). Perfect Glued Graphs at Complete Clones. Journal of Mathematics Research, 1, 25-30.

Uiyyasathian C. and Hemakul W. (2006). Planar Glued Graphs. Scientific Research (Section T), 3, 109-118.
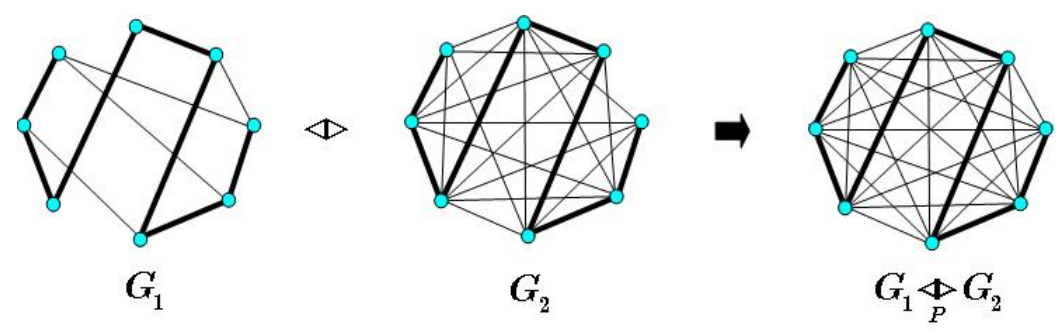

Figure 1. A glued graph with clique partition number less than those of both original graph

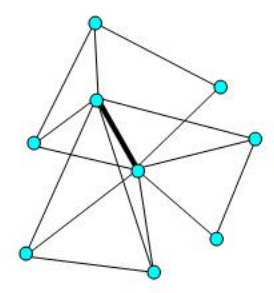

$\boldsymbol{G}$

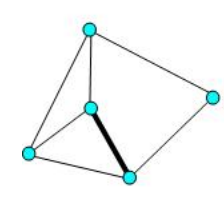

$G_{1}$

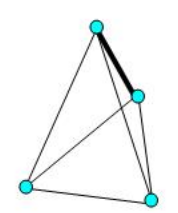

$\boldsymbol{G}_{2}$

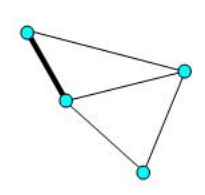

$G_{\mathbf{g}}$

Figure 2. Picture illustrating Example C 